\title{
Liquid Sloshing in a Horizontal Circular Container with Eccentric Tube under External Excitation
}

\author{
Mohammad Nezami, ${ }^{1}$ Mohammad Mehdi Mohammadi, ${ }^{1,2}$ and Atta Oveisi ${ }^{2,3}$ \\ ${ }^{1}$ Department of Mechanical Engineering, Islamic Azad University, Firoozkooh Branch, Firoozkooh, Iran \\ ${ }^{2}$ School of Mechanical Engineering, Iran University of Science and Technology, Tehran, Iran \\ ${ }^{3}$ Young Researchers and Elites Club, Islamic Azad University, Science and Research Branch, Tehran, Iran \\ Correspondence should be addressed to Atta Oveisi; atta.oveisi@gmail.com
}

Received 23 February 2014; Accepted 27 July 2014; Published 7 September 2014

Academic Editor: Mohammad Elahinia

Copyright (c) 2014 Mohammad Nezami et al. This is an open access article distributed under the Creative Commons Attribution License, which permits unrestricted use, distribution, and reproduction in any medium, provided the original work is properly cited.

Appropriate conformal mapping transformation in combination with the linear potential theory is employed to develop mathematical model for two-dimensional sloshing in horizontal circular cylindrical containers with overall eccentric hole. The tube-type tank is filled with inviscid incompressible fluid up to its half depth and subjected to lateral accelerations. A ramp-step excitation encountered in a road turning maneuver as well as real seismic event is used to simulate the lateral acceleration excitation. The resulting linear sets of ordinary differential equations are truncated and then solved numerically by employing Laplace transform technique followed by Durbin's numerical inversion pattern. The effects of excitation input time, eccentricity, and radii ratio on the hydrodynamic responses and suppression of the induced destabilizing lateral forces are examined. Limiting cases are considered and good agreements with available analytic and numerical solutions as well as the simulations performed by using a commercial FEM software package are obtained.

\section{Introduction}

Slosh refers to the splash of liquid which has a free surface inside a container that it may also undergo an undesirable motion. Practical examples consist of propellant slosh in spacecraft storage tanks, rockets, cargo slosh in battleship, road vehicles transporting liquids, and nuclear reactors. In order to model this phenomenon extensive mathematical relationships have been derived to explain liquid slosh such as [1].

Slosh is an imperative effect not only in safety of aircrafts, spacecrafts [2] but also in performance of instruments such as Earth-orbiting satellites [3,4] and attitude control system (ACS), especially for spinning satellites [5]. Recently, a wide amount of studies on liquid sloshing in horizontal cylindrical tanks with application in road and marine vehicles is done such as [6-10].

Budiansky [11] estimated the natural frequencies, mode shapes, and hydrodynamic forces in a circular cylindrical horizontal tank with partially full liquid and under transverse load excitation for the first time. McCarty and Stephens [12] calculated natural frequencies for transverse sloshing in flat cylindrical vessels with various sizes, fullness, and orientations, verifying the results presented in [11]. Moiseev and Petrov [13] measured natural frequencies with Ritz variation method for different tank geometries containing partially full liquid. They also studied the results of the horizontal circular cylindrical container in their results. McIver [14] exerted special coordinate systems to study the two-dimensional sloshing frequencies of fluid in a horizontal circular cylindrical tank filled up to an arbitrary height, reformulating the eigenvalue-sloshing problem in terms of integral equations, which were then solved numerically. Kobayashi et al. [15] found natural frequencies of sloshing for cylindrical horizontal vessel and slosh forces in two kinds of longitudinal and transverse excitation for small and large slosh wave heights experimentally. Evans and Linton [16] introduced a new semianalytical procedure which stated on expanding the velocity potential in terms of nonorthogonal bounded spatial functions to find the natural frequencies of 
sloshing in a cylindrical half full tank. Popov et al. [17] investigated the dynamic response of a partially full tank under a sudden acceleration transverse excitation to the application of road vehicle. Papaspyrou et al. $[18,19]$ used the semianalytical series-type solution to investigate the dynamic response of sloshing to half full rigid horizontal cylindrical vessels under external seismic transverse force. Dai and $\mathrm{Xu}$ [7] used the continuous coordinate transformation to analyze the sloshing liquid in circular cylindrical horizontal container with partially filled liquid. Romero et al. [6] utilized a scaled model for experimental purposes and compared the natural frequencies of different cylindrical containers with circular, elliptical, and finally generic cross section. Karamanos et al. [20] used the "impulsive-convective" decomposition method to analyze sloshing in a cylindrical horizontal circular tank with moving liquid under arbitrary horizontal ground motion. Patkas and Karamanos [21] offered a variational formulation to investigate sloshing induced from external level excitation in a cylindrical horizontal container with arbitrary level of the liquid height. Zhou et al. [22] used a finite element model for investigation of sloshing in cylindrical circular horizontal vessel. Recently, Lakis et al. [23] utilized a hybrid finite element approach combined with Sanders shell theory and classic finite element model to study the effect of free surface motion on dynamic response of a half full cylindrical circular thin anisotropic container.

Reducing the sloshing and consequent undesired results to both environmental damages and economic losses force the researchers to investigate the problem of system stability. These studies aim to consider the natural frequencies, dynamic response, and the fluid motion control for reaching the system stability. Meanwhile, in the practical problems, such as liquid storage tanks, sloshing attenuation may be achieved by covering the free surface of the fluid with a structural member, such as annular plate or disk-type obstacles named baffles. These baffles make the natural sloshing frequencies differ from the excited frequency and cause the energy to dissipate and consequently cause the reduction of sloshing amplitude [24].

A few years after the time Budiansky [11] considered the natural sloshing frequency for horizontal container, the sloshing suppression behavior in presence of the baffles was considered as an important method in sloshing suppression. Since then, numerous authors considered the effect of baffles on sloshing. For instance, Abramson [26] studied the sloshing in baffled fuel tank of space vehicles. Strandberg [25] presented a series of experimental analyses in dynamic performance and stability of sloshing in cylindrical horizontal containers with various section geometries such as circular section with different shape of baffles. He concluded that the vertical baffles are better in stability of liquid than the containers with horizontal baffles or without any baffles. Cho et al. [27] employed a coupled acoustic-structural finite element model to calculate the eigencharacteristic of an annular plate-type baffled vertical cylindrical liquid tank. Gedikli and Ergüven [24] used a variational boundary element method to investigate the sloshing problem using Hamilton method and evaluated the influence of baffle size and location on natural frequencies of upright rigid cylindrical tank. Gavrilyuk et al.
[28] applied an analytically oriented procedure to achieve the fundamental solution of linearized dynamical equation of sloshing for vertical cylindrical tank with horizontal rigid baffle. Biswal et al. [29] developed a finite element scheme based on Galerkin method and used fourth order RungeKutta to solve the nonlinear time dependent sloshing problem in an upright cylindrical tank of rigid body with rigid baffle under sinusoidal base excitation. Modaressi-Tehrani et al. [30] solved the nonlinear three-dimension transient problem of sloshing in a horizontal partially full tank with disk-type circular annular curved baffles using the FLUENT simulation software and considered the influence of baffles on stability of the tanks under input excitation. Maleki and Ziyaeifar [31] investigated the resulting damping ratio from horizontal ring and vertical blade baffles in cylindrical circular vessels using a hydrodynamic model based on Laplace's differential equation of motion. Chantasiriwan [32] used the method of fundamental solution in order to find the natural frequencies and mode shapes of liquid in rigid tanks with arbitrary shape. He also studied the results of a vertical cylindrical tank with a horizontal annular baffle. Hasheminejad and Aghabeigi [33] presented the free lateral sloshing in elliptical cylindrical half full horizontal container with a pair of longitudinal horizontal side baffles using linear potential theory. Yan et al. [34] investigated the slosh forces and moments in a partially filled container under longitudinal excitation experimentally. They also considered various tank volumes, excitations, and baffles in their results and compared them for the different input excitation and sloshing amplitude with and without baffle. Yan et al. [35] presented a two-phase flow dynamic equation of motion for sloshing liquid and solved the problem using Navier-Stokes (NS) solver. They considered various types of excitation and liquid fullness in studying transient and steady state response. They also evaluated their results with actual experimental data and FLUENT software. Pal [36] developed a two-dimensional meshless local Petrov-Galerkin procedure for the numerical simulation of local symmetric weak form of nonlinear sloshing of liquid.

This review obviously shows that there exists remarkable body of literature on liquid sloshing in upright cylindrical containers with a variety of baffle configurations. However, to the authors' best knowledge, exact theoretical or numerical solutions for free lateral liquid motion in partially filled horizontal circular cylindrical containers with overall hole seem to be absent. Faltinsen and Timokha [37] studied the two-dimensional forced liquid sloshing in a circular tank by using the multimodal method which utilizes an expansion in terms of the natural modes of free oscillations in the unforced tank. For this purpose they assumed the fluid to be an incompressible inviscid one with irrotational flow and linear free surface conditions are assumed. Takahara and Kimura [38] investigated the dynamic frequency responses of stable planar and rotary motions in a partially filled annular cylindrical tank, under a pitching excitation. They studied the response at frequency near the lowest resonant frequency. They also considered the nonlinearity of the liquid surface oscillation and the nonlinear coupling between the dominant modes and other modes in their analysis. Papaspyrou et al. $[18,19]$ tackled the problem of sloshing effects in half 


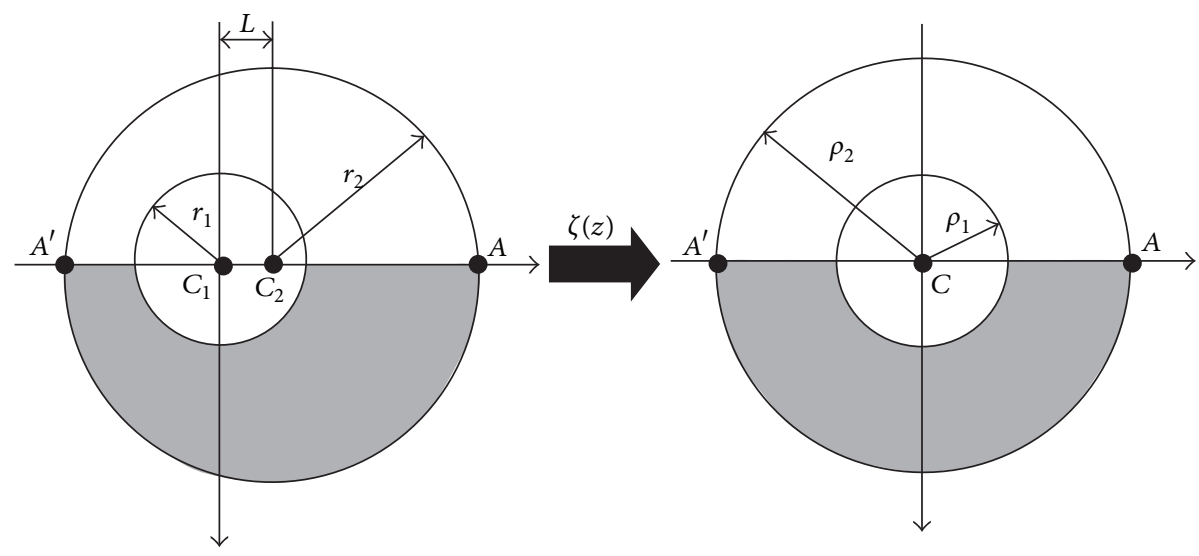

Figure 1: Problem configuration.

full horizontal cylindrical vessels, under external excitation in the direction of the longitudinal vessel axis based on a mathematical model. They calculated the hydrodynamic pressures and forces for harmonic excitation. Robu et al. [39] solved the problem of active reduction in the structural vibrations induced by the sloshing of large masses of fuel inside a partly full tank with application in an aircraft wing made of an aluminum rectangular plate with a cylindrical tip tank. Williams and Wang [40] employed a nonlinear, dispersive, dissipative shallow water theory in two horizontal dimensions to study the waves produced by the bidirectional, translational, oscillatory motion of a rectangular tank partially filled with liquid. Akyildiz [41] studied the liquid sloshing in a moving partially filled rectangular tank with a vertical baffle using a numerical algorithm based on the volume of fluid (VOF) technique. This numerical model solves the nonlinear behavior of liquid sloshing considering the complete Navier-Stokes equations in primitive variables by using finite difference approximations with the moving coordinate system. Jung et al. [42] aimed at investigating the effect of the vertical baffle height on the liquid sloshing in a laterally moving three-dimensional rectangular tank. They modeled the fluid as incompressible viscous two-phase flow in a tank of partially filled and employed the VOF method based on the finite volume method which has been utilized. Wu et al. [43] used a time-independent finite difference scheme with fictitious cell technique to analyze viscous fluid sloshing in 2D tanks with baffles. They employed Navier-Stokes equations in a moving coordinate system and mapped them onto a time-independent and stretched domain. Ali Goudarzi and Reza Sabbagh-Yazdi [44] investigated the nonlinearity in the numerical modeling of the liquid sloshing. These nonlinear terms originated from the governing equations of the fluid flow and the liquid free surface motion. Faltinsen et al. [45] classified the steady state three-dimensional resonant waves in a square-base tank by using the asymptotic modal system. They analyzed the effective frequency domains of stable steady state motions versus mean fluid depths and forcing amplitude. Marsh et al. [46] demonstrated the effect of a sloshing absorber's shape on liquid sloshing control performance of partially filled tank, numerically. For this purpose they used smoothed particle hydrodynamics (SPH) to model fluid-structure interaction of the structure/sloshing absorber system in two dimensions. Askari et al. [47] developed an analytical method to investigate the effects of a rigid internal body on bulging and sloshing frequencies and modes of a cylindrical container partially filled with a fluid using the Rayleigh quotient, Ritz expansion, and Galerkin method. Takahara et al. [48] investigated the nonlinear liquid motion originated from considering the nonlinearity of the liquid surface oscillation in a partially filled cylindrical tank with an eccentric core barrel. They also examined the effects of the eccentricity of core barrel on liquid surface oscillation, experimentally.

The main purpose of the current paper is to use the linear potential theory to fill the gap of partially filled horizontal circular cylindrical containers with overall hole. Meticulous consideration is paid to calculate the effect of overall circular cavity along the main axis of the container. It is assumed that the liquid is inviscid, incompressible, and restricted to small displacement irrotational sloshing. Also, the container is assumed to be a rigid one. The effect of viscosity is known to play a minor role in the sloshing behavior of liquid with low viscosity and tank with smooth surface [49]. The proposed model is of practical interest essentially due to its inherent value as a canonical problem in liquid sloshing dynamics.

\section{Formulation}

The geometry of the sloshing container is shown in Figure 1. The region between the two eccentric tubes in the horizontal circular tank has been filled to its half capacity with an incompressible and nonviscous liquid. As one can see in Figure 1, the inner and outer tube have the radii of $r_{1}$ and $r_{2}$ with center axis located at $c_{1}$ and $c_{2}$, respectively. Moreover, the eccentricity of the tank is taken to be $L$. The coordinates of system $(x, y)$ are chosen in the plane perpendicular to the cylinder base. The $y$-axis is in the plane of free surface, which occupies $c_{1}+r_{1} \leq a \leq c_{2}+r_{2}$ and $c_{2}-r_{2} \leq a \leq c_{1}-r_{1}$ and $x$-axis points vertically downwards through the midpoint of the free surface. In order to formulate the equation of motion 


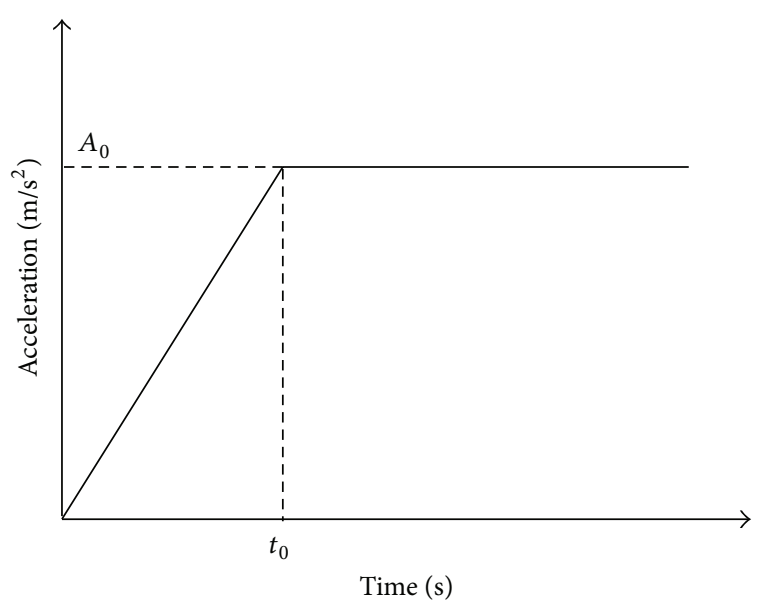

(a)

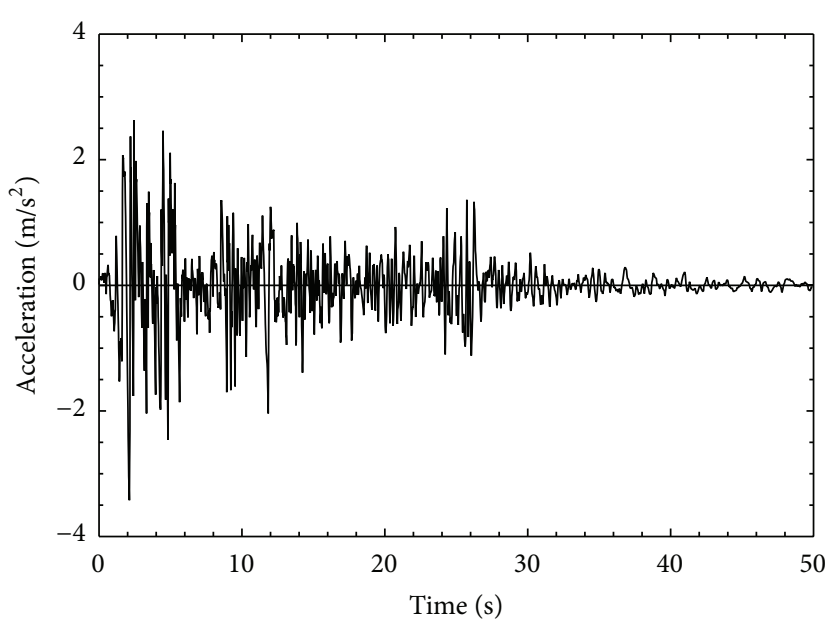

(b)

Figure 2: (a) Ideal turning movement, (b) El Centro 1940 earthquake.

an arbitrary motion, $Y(t)$, is assumed to be in the $y$-axis. Consequently, the velocity and acceleration of the container can be written as $\dot{Y}(t)$ and $\ddot{Y}(t)=A(t)$, respectively.

As mentioned before the fluid within the container is assumed to be ideal fluid. This assumption entails the existence of a velocity potential function which satisfies the Laplace equation; that is, $\nabla^{2} \Phi=0$. The free surface height is assumed to be adequately small, allowing linearization of the problem. In addition, the potential function $\Phi$ is subjected to the following linearized mixed dynamic/kinematic free surface conditions (Wang et al. [50]):

$$
\left.\frac{\partial^{2} \Phi}{\partial t^{2}}\right|_{x=0}-\left.g \frac{\partial \Phi}{\partial x}\right|_{x=0}=0
$$

with $g$ being the gravitational acceleration. Moreover, the total velocity potential may be decomposed as $\Phi=\Phi_{R}+$ $\Phi_{S}$. The first term, $\Phi_{R}$, which entirely tracks the external excitation, is directly related to the liquid rigid body motion, written as $\Phi_{R}=\dot{Y}(t) y$ [1], while the second component, $\Phi_{S}$, which indicates the sloshing component, implies the liquid motion with respect to the rigid container. By substituting the decomposition $\Phi=\dot{Y}(t) y+\Phi_{S}$ into (1), the original problem may be reduced to the solution of Laplace equation, $\nabla^{2} \Phi_{S}=0$, in a semicircular region, with the following boundary condition at the free surface:

$$
\left.\frac{\partial^{2} \Phi_{S}}{\partial t^{2}}\right|_{y=0}-\left.g \frac{\partial \Phi_{S}}{\partial x}\right|_{x=0}=-\frac{\partial^{2} \Phi_{R}}{\partial t^{2}}=-\dot{A}(t) y
$$

Also another boundary condition that has to be satisfied is the zero normal liquid velocity at the rigid container boundaries

$$
\frac{\partial \Phi_{S}}{\partial n}=0
$$

where $n$ is the normal vector to the rigid boundary. In order to satisfy boundary condition (3) on the outer surface, inner tube, and the free surface boundary conditions (2), one has to transfer the geometry of the problem into a simpler one by using the appropriate mapping. Using such a mapping will result in reformulating the problem into the standard Laplace equation of $\nabla^{2} \varphi_{s}=0$ [33]. Also, by using this method the partial differential equation will be simplified to an ordinary system of differential equations. Here, the detailed procedure of finding the solution for a container with an inner eccentric tube (see Figure 1) will be described. By using the following complex function the circular zone with inner eccentric tube of radius $r_{1}$ and outer radius of $r_{2}$ in $z$-plane will be mapped onto an annular tube with inner and outer radius of $\rho_{1}$ and $\rho_{2}$ on $w$-plane, respectively. Consider

$$
w=v-i u=-\rho e^{i \theta}=\frac{z}{1+\alpha z}, \quad z=y-i x,
$$

where $\alpha, \rho_{1}$, and $\rho_{2}$ are geometric constants which can be calculated as

$$
\begin{aligned}
& a=\frac{L}{\sqrt{\left(\left(r_{1}^{2}-r_{2}^{2}\right)^{2}-2 L\left(r_{1}^{2}+r_{2}^{2}\right)+L^{4}\right)}}, \\
& \rho_{1}=\frac{\sqrt{1+4 r_{1}^{2} a^{2}}-1}{2 r_{1} a^{2}}, \quad \rho_{2}=\frac{\sqrt{1+4 r_{2}^{2} a^{2}}-1}{2 r_{2} a^{2}} .
\end{aligned}
$$

It is clearly seen from Figure 2(b) that the outer and inner eccentric circles are mapped onto the two concentric circles with $\rho_{2}$ and $\rho_{1}$ radii, respectively. Meanwhile, the free surface will be mapped onto the boundaries $\theta= \pm \pi / 2$ in the $w$-plane. The solution of Laplace equation $\varphi_{s}$ which satisfies both inner and outer wall boundary conditions can be written in form of series as

$$
\Phi^{s}=\sum_{n=0}^{\infty} \varphi_{n(t)} \cos \left[\mu_{n} \ln \left(\frac{\rho}{\rho_{2}}\right)\right] \frac{\sinh \left(\mu_{n} \theta\right)}{\sinh \left(\mu_{n}(\pi / 2)\right)},
$$

in which $\mu_{n}=n \pi / \ln \left(\rho_{1} / \rho_{2}\right)$. It should be noted that the only mode shapes that are considered in the solution are 
antisymmetric modes. This is because of the fact that the symmetric mode shapes under transverse loading will not be excited [11]. Furthermore, by using the chain rule in differentiation, one can rewrite the free surface boundary condition (2) in the $w$-plane as

$$
\gamma(\rho) \frac{\partial^{2} \Phi}{t^{2}}-\left.g \frac{\partial \Phi}{\partial \theta}\right|_{ \pm \pi / 2}=-\dot{A}(t) y\left(\rho, \pm \frac{\pi}{2}\right),
$$

where

$$
\gamma(\rho)=\frac{\mp \rho}{(1 \mp a \rho)^{2}} .
$$

It should be noted that the $\gamma(\rho)$ function contains the geometric parameters. By substituting (6) in (7) one will get

$$
\begin{aligned}
& \pm \sum_{n=0}^{\infty} \ddot{\varphi}_{n(t)} \cos \left[\mu_{n} \ln \left(\frac{\rho}{\rho_{2}}\right)\right] \\
& \quad-g \sum_{n=0}^{\infty} \mu_{n} \varphi_{n(t)} \cos \left[\mu_{n} \ln \left(\frac{\rho}{\rho_{2}}\right)\right] \\
& \quad \times \operatorname{coth}\left(\mu_{n} \frac{\pi}{2}\right)=-\gamma(\rho) \dot{A}(t) y\left(\rho, \pm \frac{\pi}{2}\right),
\end{aligned}
$$

where $y(\rho, \pm \pi / 2)$ will be calculated by substituting $x=0, \theta=$ $\pm \pi / 2$ in (4) and solving the resulting equation with respect to $y$. Then, by using Euler orthogonal expansion functions with respect to the weighting function of $1 / \rho$, the product functions of $\gamma(\rho) y(\rho)$ can be calculated as

$$
-\gamma(\rho) y(\rho)=\sum_{n=0}^{\infty} f_{n} \cos \left[\mu_{n} \ln \left(\frac{\rho}{\rho_{2}}\right)\right],
$$

where

$$
f_{n}=-\frac{\int_{\rho_{1}}^{\rho_{2}}(1 / \rho) \gamma(\rho) y(\rho) \cos \left[\mu_{n} \ln \left(\rho / \rho_{2}\right)\right] d \rho}{\int_{\rho_{1}}^{\rho_{2}}(1 / \rho) \cos ^{2}\left[\mu_{n} \ln \left(\rho / \rho_{2}\right)\right] d \rho} ;
$$

by utilizing expansion (11) in (9), (12) can be written as

$$
\begin{aligned}
& \pm \gamma(\rho) \sum_{n=0}^{\infty} \ddot{\varphi}_{n(t)} \cos \left[\mu_{n} \ln \left(\frac{\rho}{\rho_{2}}\right)\right] \\
& \quad-g \sum_{n=0}^{\infty} \mu_{n} \varphi_{n(t)} \cos \left[\mu_{n} \ln \left(\frac{\rho}{\rho_{2}}\right)\right] \operatorname{coth}\left(\mu_{n} \frac{\pi}{2}\right) \\
& =\dot{A}(t) \sum_{n=0}^{\infty} f_{n} \cos \left[\mu_{n} \ln \left(\frac{\rho}{\rho_{2}}\right)\right] .
\end{aligned}
$$

Then, by multiplying both sides of (12) in $(1 / \rho) \cos \left[\mu_{m}\right.$ $\left.\ln \left(\rho / \rho_{2}\right)\right]$ and integrating both sides in the interval $\left[\rho_{1}, \rho_{2}\right]$ the results will be as follows:

$$
\pm \sum_{n=0}^{\infty} \ddot{\varphi}_{n(t)} M_{m n}-\sum_{n=0}^{\infty} K_{m n} \varphi_{n}=\dot{A}(t) \sum_{n=0}^{\infty} f_{n} \delta_{m n}
$$

where

$$
\begin{gathered}
M_{m n} \\
=\frac{\int_{\rho_{1}}^{\rho_{2}}(1 / \rho) \gamma(\rho) \cos \left[\mu_{n} \ln \left(\rho / \rho_{2}\right)\right] \cos \left[\mu_{m} \ln \left(\rho / \rho_{2}\right)\right] d \rho}{\int_{\rho_{1}}^{\rho_{2}}(1 / \rho) \cos ^{2}\left[\mu_{n} \ln \left(\rho / \rho_{2}\right)\right] d \rho} \\
K_{m n}=g \mu_{n} \operatorname{coth}\left[\mu_{n}\right] \delta_{m n}, \quad K_{00}=\frac{2}{\pi}
\end{gathered}
$$

$n=0,1, \ldots$. Finally, with truncation of (13), one will get

$$
\mathbf{M} \ddot{\varphi}(t)-\mathbf{K} \varphi(t)=\dot{A}(t) \mathbf{f},
$$

in which $\mathbf{M}=\left[M_{m n}\right]$ is an $N \times N$ symmetric square matrix, $\mathbf{K}=\operatorname{Dig}\left[k_{m, m}\right]$ is $N \times N$ diagonal matrix, $\boldsymbol{\varphi}(t)=$ $\left[\varphi_{1}(t), \varphi_{2}(t), \ldots, \varphi_{N}(t)\right]^{T}$ is vector of unknown generalized coordinates, and $\mathbf{f}=\left[f_{1}, f_{2}, \ldots, f_{N}\right]^{T}$ is the external excitation coefficient vector. Also, $f, M$, and $K$ describe the external excitation vector, mass matrix, and stiffness matrix, respectively. And $N$ is the truncation constant. The transient response of the coupled system will be calculated by solving (15). For this purpose and in order to transfer the results from Laplace domain to time domain the inverse Laplace based on Durbin method will be utilized. By assuming the initial condition to be zero, that is, $\varphi(0)=\dot{\varphi}(0)=A(0)=0$, and implementing Laplace transformation in (15) one will get

$$
\left(s^{2} \mathbf{M}-\mathbf{K}\right) \overline{\boldsymbol{\varphi}}(s)=s \bar{A}(s) \mathbf{f},
$$

where $\bar{\varphi}(s)$ and $\bar{A}(s)$ present the transformed relations of $\varphi(t)$ and $A(t)$, respectively. At this point, the generalized coordinate vector $\varphi(t)$ can be solved using (16) as

$$
\boldsymbol{\varphi}(t)=\mathscr{L}^{-1}\left[s \bar{A}(s)\left(s^{2} \mathbf{M}-\mathbf{K}\right)^{-1} \mathbf{f}\right]
$$

Moreover, by using (6) together with kinematic free surface condition, $\partial \eta / \partial t=(1 / \gamma(\rho))\left(\partial \varphi_{s} / \partial \rho\right)$, in which $\eta(\rho, t)$ presents the free surface height, the following expansion can be written:

$$
\begin{gathered}
\eta(\rho, t)=\frac{-1}{\gamma(\rho)}\left(\frac{2}{\pi} \eta_{0(t)}+\sum_{n=1}^{\infty} \eta_{n(t)} \mu_{n} \operatorname{coth}\left(\mu_{n} \frac{\pi}{2}\right)\right. \\
\left.\times \cos \left[\mu_{n} \ln \left(\frac{\rho}{\rho_{2}}\right)\right]\right),
\end{gathered}
$$

where $\eta_{n}(t)=\int_{0}^{t} \varphi_{n}(t) d t$. Furthermore, by using the Laplace transformation properties, that is, $\bar{\eta}_{n}(s)=(1 / s) \bar{\varphi}_{n}(t)$ (Hildebrand [51]), the transformed equations of motion (16) lead to

$$
\left(s^{2} \mathbf{M}-\mathbf{K}\right) \overline{\boldsymbol{\eta}}(s)=\bar{A}(s) \mathbf{f},
$$


which can readily be inverted as follows:

$$
\boldsymbol{\eta}(t)=\mathscr{L}^{-1}\left[\bar{A}(s)\left(s^{2} \mathbf{M}-\mathbf{K}\right)^{-1} \mathbf{f}\right]
$$

where $(t)=\left[\eta_{1}(t), \eta_{2}(t), \ldots, \eta_{N}(t)\right]^{T}$. By using the modal coefficients (20) in (18) one can calculate the free surface height in the $w$-plane. Finally, in order to map the results onto the physical original $z$-plane, (4) will be used. The hydrodynamic pressure can be calculated on container's wall and inner tube by substituting the expansion series (6) in $\varphi=x(t) x+\varphi_{s}$ and using linear Bernoulli equation (1) at $\rho=\rho_{1}, \rho_{2}$, respectively, as

$$
\begin{aligned}
& P_{\text {wall }}=P\left(\rho_{2}, \theta, t\right)=-\left.\rho \frac{\partial \Phi}{\partial t}\right|_{\rho=\rho_{2}} \\
& =-\rho\left\{A(t) y\left(\rho_{2}, \theta\right)+\frac{2 \theta}{\pi} p_{0}(t)\right. \\
& \left.+\sum_{n=1}^{\infty} p_{n}(t) \frac{\sinh \left(\mu_{n} \theta\right)}{\sinh \left(\mu_{n} \frac{\pi}{2}\right)}\right\} \\
& P_{\text {tube }}=P\left(\rho_{1}, \theta, t\right)=-\left.\rho \frac{\partial \Phi}{\partial t}\right|_{\rho=\rho_{1}} \\
& =-\rho\left\{A(t) y\left(\rho_{1}, \theta\right)+\frac{2 \theta}{\pi} p_{0}(t)\right. \\
& \left.+\sum_{n=1}^{\infty} p_{n}(t) \cos \left[\mu_{n} \ln \left(\frac{\rho_{1}}{\rho_{2}}\right)\right] \frac{\sinh \left(\mu_{n} \theta\right)}{\sinh \left(\mu_{n} \frac{\pi}{2}\right)}\right\},
\end{aligned}
$$

in which $\rho, P_{\text {wall }}$, and $P_{\text {tube }}$ are the density of the fluid and pressure on containers wall and inner tube, respectively (see Figure 1). Also, by implementing derivative relation of Laplace transform, $\bar{p}_{n}(s)=s \bar{\varphi}_{n}(s)$, in (12) and using $\bar{p}_{n}(s)=$ $s \bar{\varphi}_{n}(s)$, the pressure coefficient vector will be achieved as follows:

$$
\mathbf{P}(t)=\mathscr{L}^{-1}\left[s^{2} \bar{A}(s)\left(s^{2} \mathbf{M}-\mathbf{K}\right)^{-1} \mathbf{f}\right]
$$

where $\mathbf{P}(t)=\left[p_{1}, p_{2}, \ldots, p_{N}\right]^{T}$. The horizontal force per length of the tank calculated by integrating the dynamic pressure (21) in the form

$$
F_{y}(t)=\int\left(P_{\text {wall }}+P_{\text {tube }}\right) d x=F_{R}(t)+F_{S}(t),
$$

where $F_{R}(t)=-\rho A(t) \int\left(y\left(\rho_{1}, \theta\right)+y\left(\rho_{2}, \theta\right)\right) d x=-\rho\left[(\pi / 2)\left(r_{2}^{2}-\right.\right.$ $\left.\left.r_{1}^{2}\right)\right] A(t)=-m A(t)$ presents the rigid body motion of the fluid and $F_{S}(t)=-2 \rho \sum_{n=0}^{\infty} G_{n} p_{n}(t)$, and

$G_{n}$

$$
= \begin{cases}\int_{0}^{\chi_{0}} \frac{2 \theta}{\pi}\left[\left.\frac{\partial x}{\partial \theta}\right|_{\rho=\rho_{1}}+\left.\frac{\partial x}{\partial \theta}\right|_{\rho=\rho_{2}}\right] d \theta, & (n=0), \\ \int_{0}^{\pi / 2} \cos \left[\mu_{n} \ln \left(\frac{\rho_{1}}{\rho_{2}}\right)\right] & \\ & \times\left.\frac{\sinh \left(\mu_{n} \theta\right)}{\sinh \left(\mu_{n}(\pi / 2)\right)} \frac{\partial x}{\partial \theta}\right|_{\rho=\rho_{1}} d \theta \\ & +\left.\int_{0}^{\pi / 2} \frac{\sinh \left(\mu_{n} \theta\right)}{\sinh \left(\mu_{n}(\pi / 2)\right)} \frac{\partial x}{\partial \theta}\right|_{\rho=\rho_{2}} d \theta, \quad(n=1,2,3, \ldots),\end{cases}
$$

which states for fluid liquid sloshing. Also, $m$ is the mass of the fluid per unit length of the tank. It should be noted that in the simulations only the horizontal force which can cause the instability of the tank is investigated.

\section{Numerical Example}

In this section the transient response of the presented model is investigated for various key parameters such as radii ratios of $r_{1} / r_{2}$ and eccentricity ratios $e$ under different lateral excitation. Realizing the large number of parameters involved here while keeping in view our computing hardware limitations, we will confine our attention to a particular problem. Accordingly, it is assumed that the circular tank $\left(r_{2}=1\right)$ with half full water $\left(\rho=1000 \mathrm{~kg} / \mathrm{m}^{3}\right)$ is excited under two external excitations as shown in Figure 2(a).

Ideal turning movement (Figure 2(a)):

$$
A(t)= \begin{cases}\frac{A_{0} t}{t_{0}} & \left(t \leq t_{0}\right) \\ A_{0} & \left(t>t_{0}\right)\end{cases}
$$

El Centro 1940 earthquake (Figure 2(b)): http://www.vibrationdata.com/elcentro_NS.dat.

It is also assumed that the final steady value of $A_{0}$ is equal to $A_{0}=v^{2} / R=0.3 \mathrm{~g}$ with $v$ and $R$ being the speed of tank and maneuver radius, respectively. A symbolic software (Mathematica) is utilized in order to calculate the physical parameters: $f_{n}, M_{n m}, K_{m m}, \varphi_{s}, \eta, P, F_{s}$, and $F_{R}$. Also, for numerical integration the $N$ integrate function is used. Moreover, as it is mentioned before for numerical Laplace inverse the Durbin method is used to calculate $\varphi(t), \eta(t)$, and $P(t)$ in (17), (20), and (22), respectively. For the lateral ground acceleration (Figure 2(b)), the built-in Mathematica function "NDSolve" was used for solving the system of ordinary differential equations (15) and subsequently obtaining $\mathbf{P}(t)=$ $\partial \varphi(t) / \partial t$. The convergences of the numerical calculations are guaranteed by increasing the constant $\bar{N}_{\max }=700$ and truncation constant $N_{\max }=30$. Before presenting the results, the validation of the presented model is investigated. For this purpose, the simulation results are compared to those presented by Strandberg [25] for the maximum steady state 

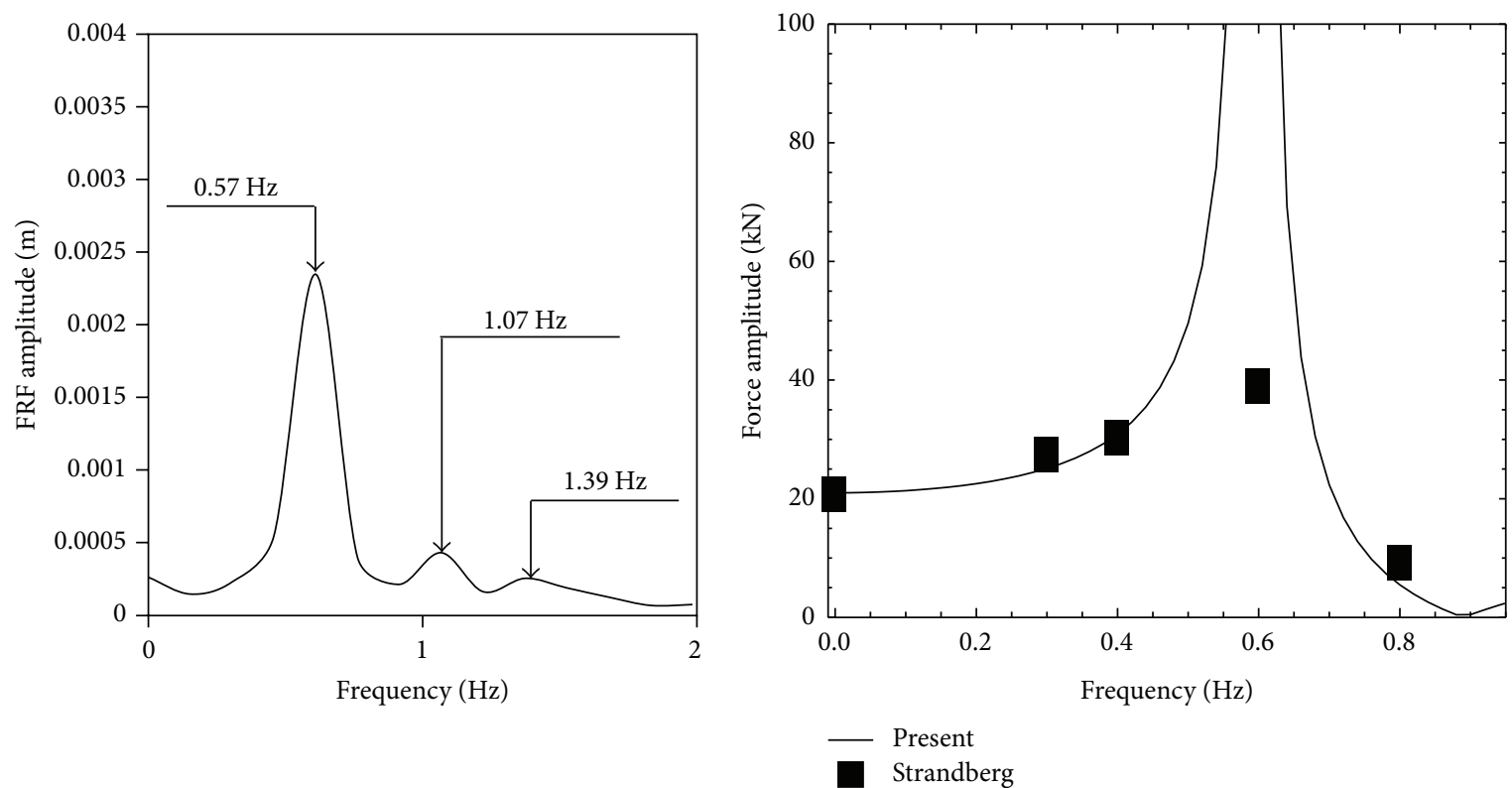

(a)
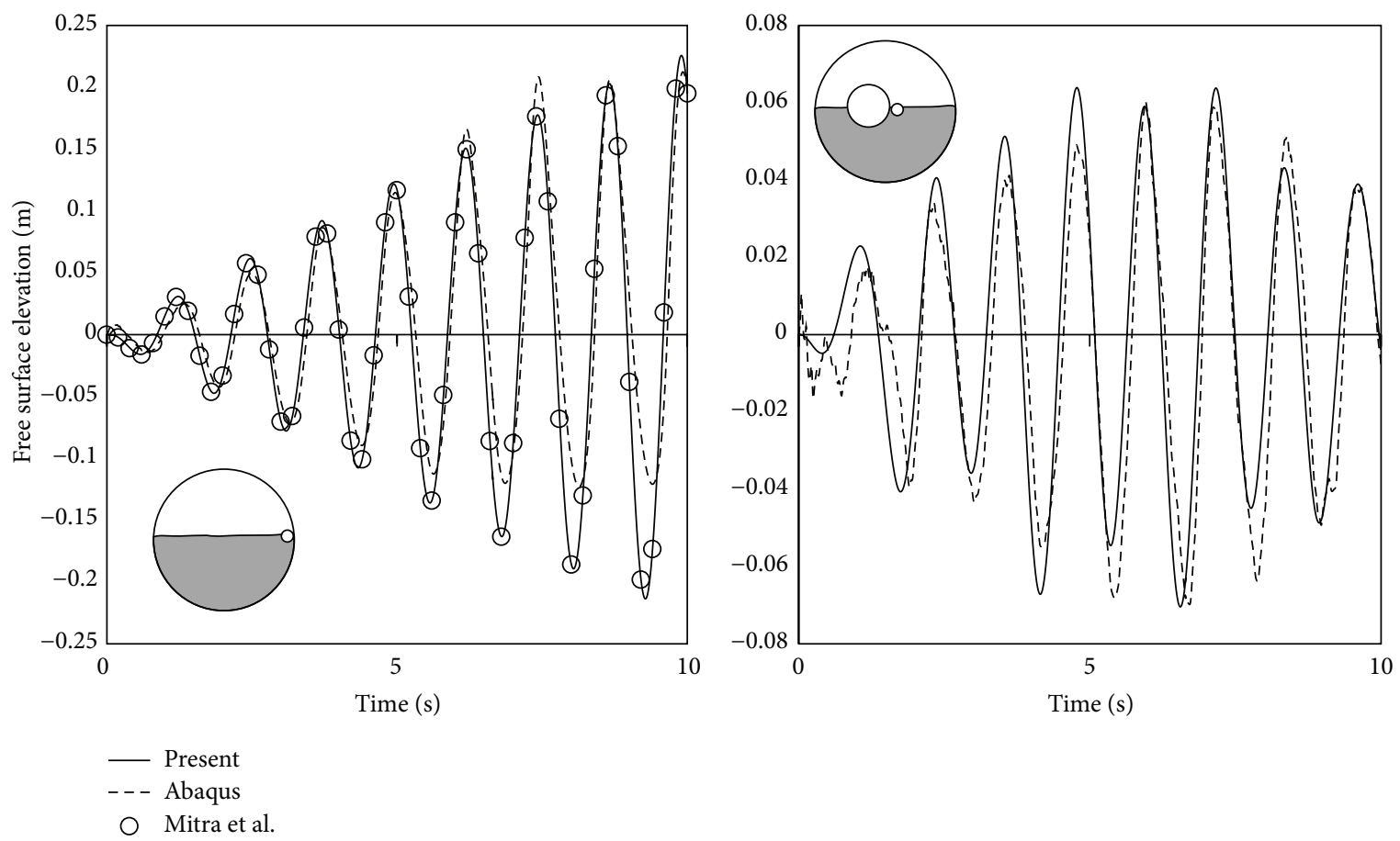

(b)

FigURE 3: (a) Verification with results offered by Strandberg [25], (b) validated results with Abaqus.

pressure in a circular half full oil tank without inner tube $\left(\rho=920 \mathrm{~kg} / \mathrm{m}^{2}, r_{1} / r_{2}=0.01\right.$, and $\left.r_{2}=0.95\right)$ under harmonic acceleration excitation. For this purpose, by derivation of (15) with respect to time and replacing $P(t)=P(\omega) \sin \omega t$ and $A(t)=A_{0} \sin \omega t$ with $A_{0}=2.1\left(\mathrm{~m} / \mathrm{s}^{2}\right)$, the maximum steady state pressure will result as $P(\omega)=-\omega^{2} A_{0}\left(K-\omega^{2} M\right)^{-1} f$, and by substituting this in (23) the steady state pressure amplitude can be easily calculated. The results as depicted in second subplot of Figure 3(a) present a good agreement with those offered by Strandberg [25]. As one can see, the results diverge around the natural frequencies which are because of the damping effect and nonlinearities that are available in physical system but not considered in the presented model. Moreover, the transient free surface height of the presented model, for circular tank without inner tube near the tank wall $\left(\rho=1000 \mathrm{~kg} / \mathrm{m}^{3}, \gamma_{1} / \gamma_{2}=0.01\right.$, and $\left.\gamma_{2}=0.5\right)$, with lateral 
displacement excitation of $y(t)=-0.01 \sin 5 t$, is compared to the results obtained from commercial finite element code (Abaqus). An effective method for modeling inviscid fluids in Abaqus/Explicit is to use a linear $U_{s}-U_{p}$ equation of state for the bulk response. The bulk modulus is chosen two or three orders of magnitude less than the actual bulk modulus and the water will still behave as an incompressible medium. For this problem the linear $U_{s}-U_{p}$ equation of state is used with a wave speed of $45.103 \mathrm{~m} / \mathrm{s}$ and density of $1000 \mathrm{~kg} / \mathrm{m}^{3}$. This wave speed corresponds to a bulk modulus of $2.07 \mathrm{MPa}$ (three orders of magnitude less than the actual bulk modulus of water, 2.07 GPa). Adaptive meshing is utilized in order to model the liquid domain. Moreover, the sliding boundary condition is employed for defining the contact face because the shearing, which is caused by the sloshing motion, is zero. The frequency and the intensity of adaptive meshing have to be increased to offer a smooth mesh. The value of the frequency parameter on the adaptive mesh option is reduced from the default of 10 to 5 , and the value of the mesh sweeps parameter is increased from the default of 1 to 3. The 4-node bilinear plane strain quadrilateral element is used for FEM analysis. Results as shown in the first subplot of Figure 3(b) have acceptable verification. Also, in this figure a good convergence is shown with the one presented by Mitra et al. [52]. In addition, the results for a circular tank with inner tube $\left(r_{1} / r_{2}=0.2, \gamma_{2}=1\right.$, and $\left.L=0.32\right)$ are compared to the one obtained from Abaqus with the same lateral excitation around inner tube wall. The second subplot of Figure 3(b) shows good agreement with results obtained from FEM. Finally, the transient free surface response of a circular tank without inner tube with impulsive excitation $\left(\gamma_{1} / \gamma_{2}=0.01\right.$ and $\left.\gamma_{2}=1\right)$ is calculated. Then, as shown in the first subplot of Figure 3(a) the frequency domain response (FRF) of the model is evaluated by implementing fast Fourier transformation (FFT) in the time domain response. The picks as presented in Figure 3(a) are in a good agreement with the three first nonsymmetrical natural frequencies that are presented by McIver [14]. Table 1 presents the first four normalized antisymmetric/symmetric sloshing frequencies $\left(\omega^{2} r_{2} / g\right)$ of the horizontal circular tanks with eccentric tube, for the limiting case of a nearly unbaffled $\left(r_{1} / r_{2} \approx 0\right)$ constant outer radius vessel $\left(r_{2}=1 \mathrm{~m}\right)$, with the aim of matching the results calculated by the first three columns of levels [14] for the sloshing frequencies in a partially filled (unbaffled) circular tank at selected liquid fill level $(d / c=1)$. Note that the natural sloshing frequencies were obtained by direct treatment of the eigenvalue problem $\left(\mathbf{K}+\omega^{2} \mathbf{M}\right) \widetilde{\boldsymbol{\varphi}}=0$. The outcome as displayed in Table 1 exhibits good agreements with the data provided in Table 1 of McIver [14].

The first column of Figure 4 shows variations of the antisymmetric and symmetric nondimensional eigenvalues $\left(\lambda r_{2}\right)$ for variations of radius ratios of $r_{1} / r_{2}$ in different mode shapes at the specified eccentricities. However, the effect of variation of the eccentricity for specified radius ratios is shown in second column of Figure 4 . The most important observations are as follows. First, as it is reflected in Figure 4, the eigenproblem of (15) has a trivial symmetric solution of constant $\Phi=0$ which can be considered as
TABLE 1: Validation of the natural sloshing frequencies.

\begin{tabular}{cccccc}
\hline \multicolumn{5}{c}{$r_{1} / r_{2}=0.01$} \\
& \multicolumn{5}{c}{$e=0$} \\
$n$ & McIver [14] & Present & $n$ & McIver [14] & Present \\
& A-sym & A-sym & & Sym & Sym \\
\hline 1 & 1.35573 & 1.35475 & 2 & 3.03310 & 3.0887 \\
3 & 4.65105 & 4.64375 & 4 & 6.23920 & 6.3492 \\
5 & 7.81976 & 7.8002 & 6 & 9.39668 & 9.5617 \\
7 & 10.9718 & 10.9562 & 8 & 12.5457 & 12.7668 \\
\hline
\end{tabular}

the first symmetric mode. It is also clear that frequency of the fundamental antisymmetric mode decreases by increasing $r_{1} / r_{2}$ while the higher eigenvalues show completely different trends. Another observation associated with the water waves is existence of a critical value of $r_{1} / r_{2}$ for each antisymmetric mode, after which the antisymmetric nondimensional frequency merges with its lower symmetric mode. In other words it can be stated that the symmetric modes are lower bounds of the antisymmetric ones. Also, as one can see this critical value decreases as the eccentricity increases. An important distinction between variation of antisymmetric and symmetric frequencies is that the eigenvalues of the higher antisymmetric modes are not monotonic while their symmetric counterparts increase monotonically. However, this effect decreases for higher values of eccentricity.

In the following, the dynamical characteristics of the system will be evaluated for different geometrical constants. Moreover, for demonstrating the transient responses the following transformation is used to present the results dimensionless: $\widetilde{F}_{x}=F_{x} / m A_{0}, \widetilde{t}=t \sqrt{g / r_{2}}$. The simulations are done for the different radius ratios $\left(c=r_{1} / r_{2}=0.3,0.5,0.8\right)$, eccentricities $(e=0,0.5,0.8)$, and input dimensionless time $\left(\widetilde{t_{0}}=0.01,1,2.5,5\right)$.

It should be noted that due to the linear nature of the model the magnitude of the dimensionless force is not a function of the $A_{0}=0.3 \mathrm{~g}$ [53]. As it can easily be seen in Figure 5, after reaching a steady acceleration, the lateral forces continue to oscillate around the equilibrium point without any damping (ideal liquid). As a result the induced vibrating forces under oscillatory excitations, especially for sudden accelerations, cannot be ignored. Meanwhile, the vibrating amplitude decreases as times goes on. The first case $\left(\widetilde{t_{0}}=0.01\right)$ demonstrates sudden acceleration excitation which caused the system to oscillate with the maximum sloshing magnitude which should be prevented in liquid moving vehicles.

It is clear that as the radius ratio increases the free surface length decreases and as a result $F_{s}$ (the sloshing force) decreases. However, by considering nondimensionless force $F_{s} / m A_{0}$, as $r_{1}$ increases $m$ decreases. And, finally, the amplitude of the induced sloshing force enhances at first and then reduces with respect to amplifying radius ratio. Another observation is that by increasing the eccentricity constant $e$ the pressure on the tank wall increases which is a result of increasing the liquid motion ability (see Figure 5). Also, by increasing (decreasing) the radius ratio simultaneously, the effect of eccentricity on pressure profile shrinks (increases) because the free surface area lessens. Moreover, increasing 

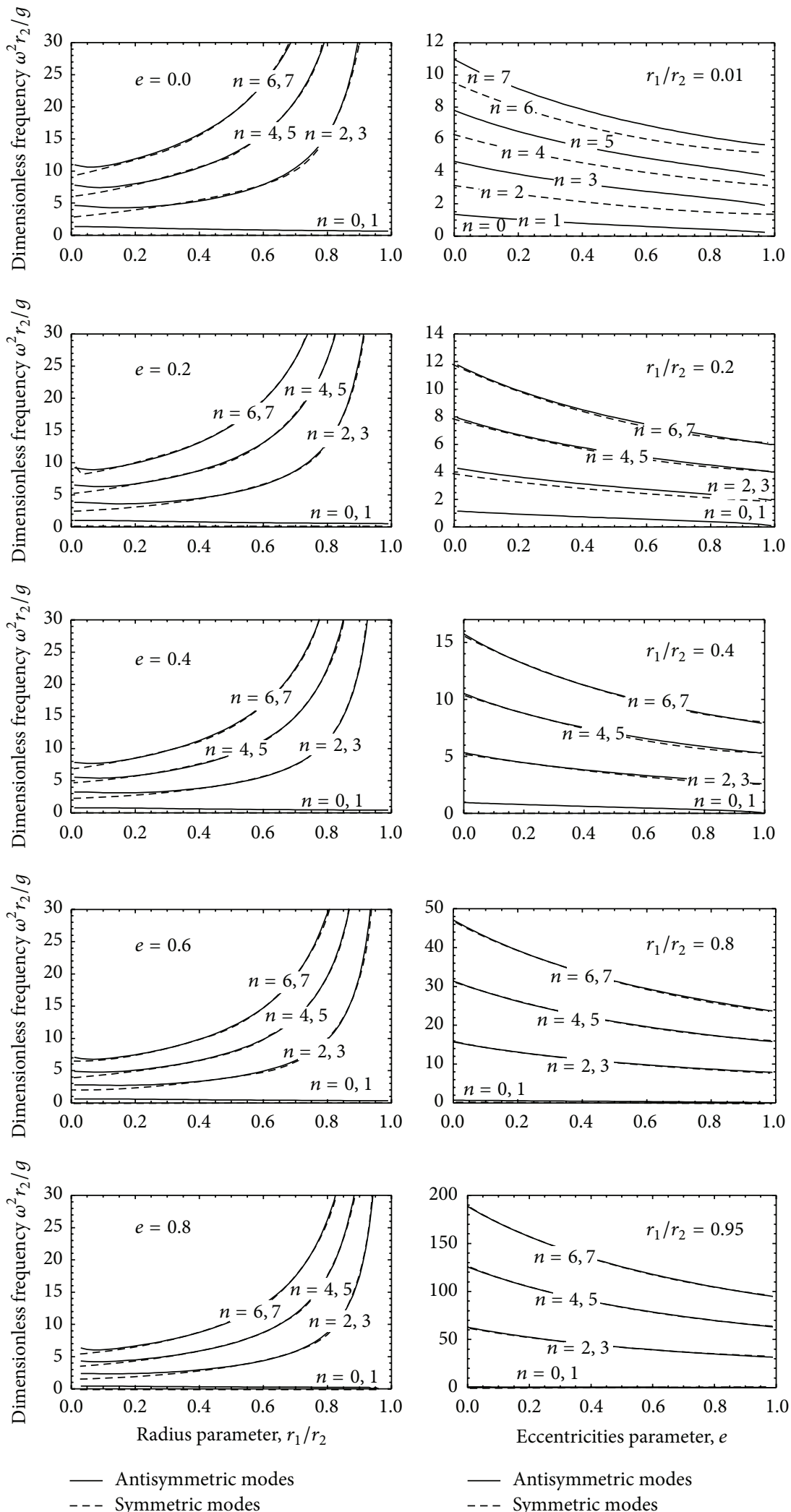

FIGURE 4: Variation of the wave motion frequencies with radii ratio and eccentricity parameter. 

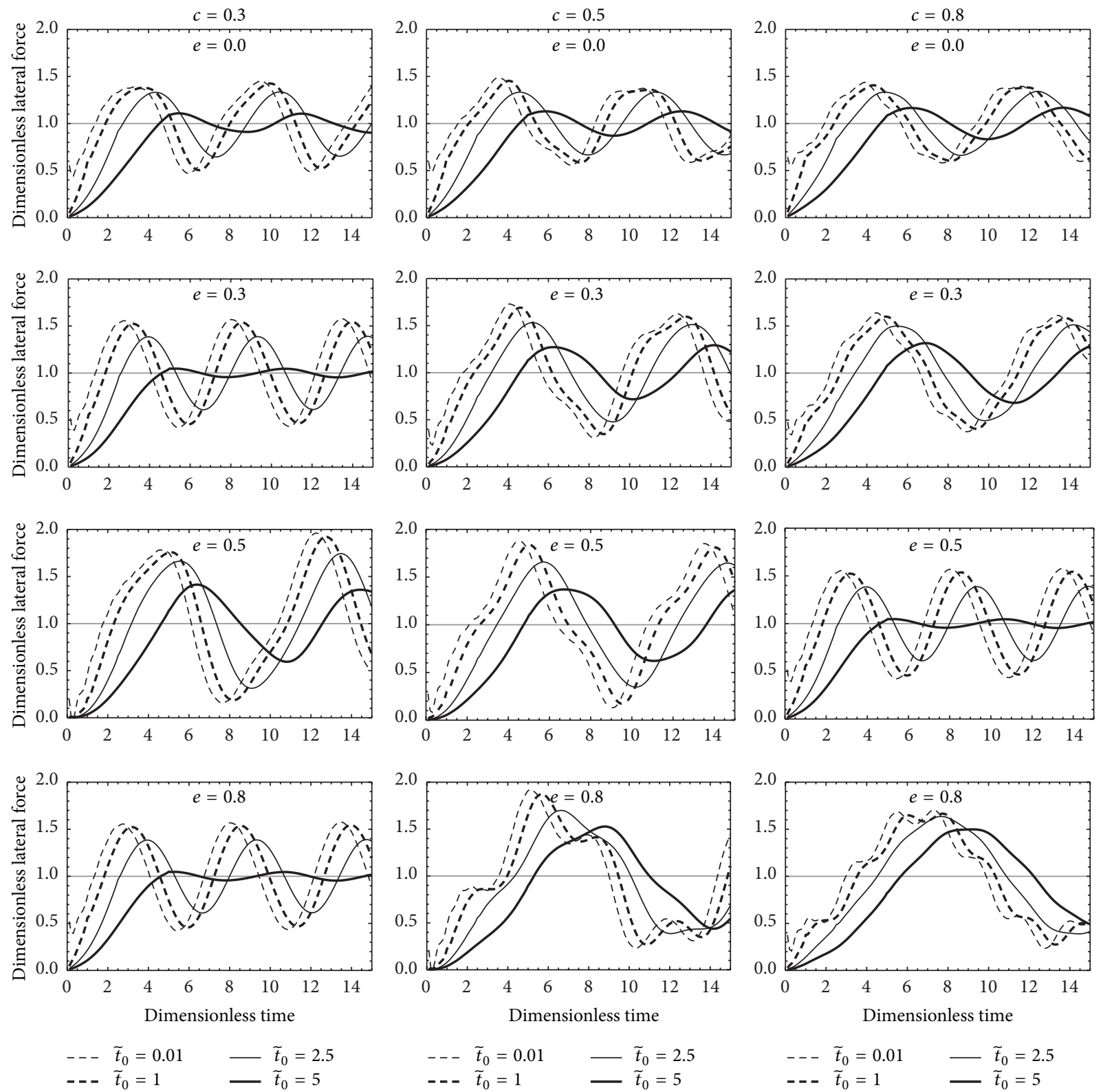

FIGURE 5: The lateral forces oscillation around the equilibrium point under oscillatory excitations.

the radius ratio will result in decreasing the first natural frequency unlike the higher order mode shapes. As a result, by increasing the radius ratio the sloshing frequency decreases (see Figure 5). This is because of the fact that the first mode shape is the dominant one in the transient sloshing response. With the same logic, increasing $e$ will result in decreasing all natural frequencies and sloshing frequency. Note that, in Figure 5 , as $t_{0}$ decreases to the minimum simulated value of $\left(t_{0}=0.01\right)$, the external excitation at initial time changes from ramp to a step so we have

$$
\widetilde{A}(s)= \begin{cases}\frac{1}{s} & t_{0} \longrightarrow 0 \\ \frac{1}{s^{2}} & t_{0} \gg 0 .\end{cases}
$$

If that is the case, having in mind (22) and $f\left(0^{+}\right)=$ $\lim _{s \rightarrow \infty} s F(s)$ in the Laplace domain, the initial pressure response will be as follows:

$$
\mathbf{P}\left(0^{+}\right)= \begin{cases}\lim _{s \rightarrow \infty}\left(\frac{s^{3} \times 1 / s \times \mathbf{f}}{s^{2} \mathbf{M}-\mathbf{K}}\right)=\mathbf{M}^{-1} \mathbf{f} & t_{0} \longrightarrow 0 \\ \lim _{s \rightarrow \infty}\left(\frac{s^{3} \times 1 / s^{2} \times \mathbf{f}}{s^{2} \mathbf{M}-\mathbf{K}}\right)=\mathbf{0} & t_{0} \gg 0 .\end{cases}
$$

One can conclude that, in Figure (5), the time response for $t_{0}=0.01$ will not start from zero and it has an initial value. Dependence of $\gamma(\rho)$ on $c$ and $e$ leads to dependence of vector $f$ on these parameters, so initial value of force changes by changing $c$ and $e$. It makes sense that a sudden increase in actuation causes a sudden increase in force. 

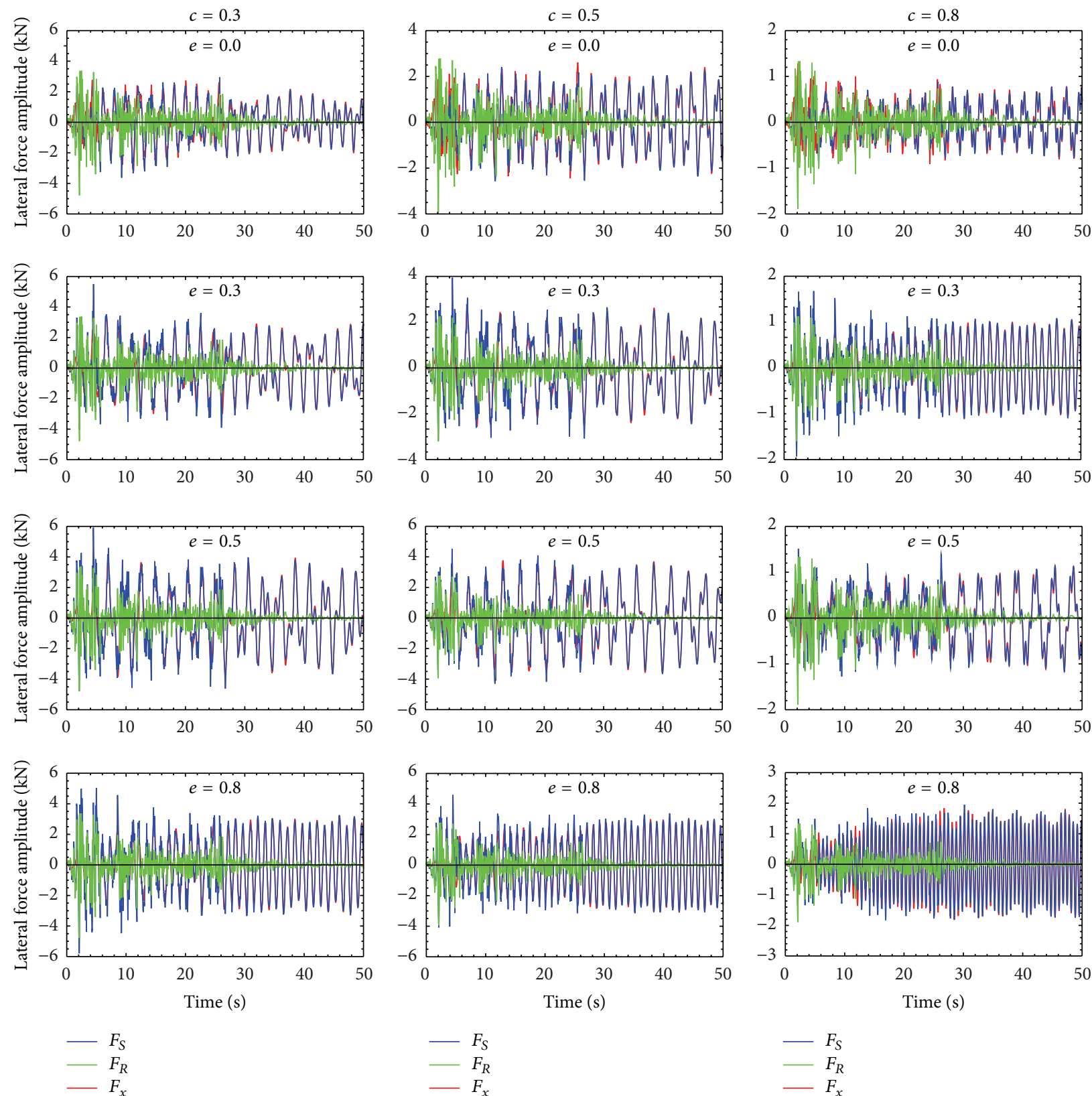

FIgURE 6: Time histories of the calculated forces of rigid motion, sloshing forces, and total forces.

Figure 6 demonstrates the time histories of the calculated forces of rigid motion, sloshing forces, and total forces $\left(F_{R}, F_{s}\right.$, and $\left.F_{x}\right)$ for seismic excitation (El Centro 1940; see Figure 2(b)) of different radius ratios and eccentricity constants. The most important observations are as follows.

For $0<t<5 \mathrm{sec}$, without considering the geometry of the problem, the amplitude of the sloshing and $F_{s}(t)$ is very small and the rigid body mode $\left(F_{R}(t)\right)$ plays the major role in the dynamic lateral response. For $5<t<35 \mathrm{sec}$, the sloshing force component increases in counteraction with the rigid body mode. However, in the cases where the radius ratio is high but the eccentricity is negligible, the rigid body mode will again dominate the sloshing force which leads to decreasing the sloshing free surface. For final time period, for $35<t$ sec, irrelevant of geometry of the problem, the sloshing forces due to the rigid body motion diminish because of significant reduction in excitation amplitude and as a result the sloshing forces play the important role in dynamic response of the hydrodynamic system. In other words, the impulsive characteristic of the hydrodynamic response is suppressed and the system response becomes convective. Finally, it should be noted that, at high radius ratios, the effect of eccentricity becomes bolder in the transient response. For instance, in high eccentricity ratios the dropping sloshing frequency is because of the excitation of the higher order mode shapes that is not the issue for low radius ratios.

Furthermore, Figure 7 visualizes the typical pressure distributions acting on the tank wall as well as tube and free 


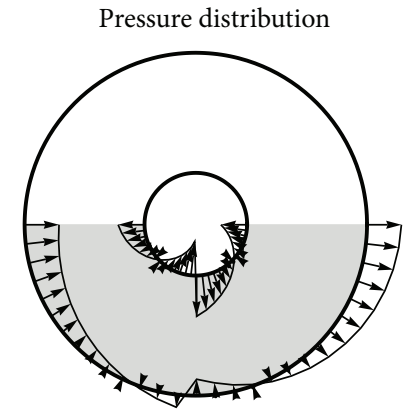

(a)

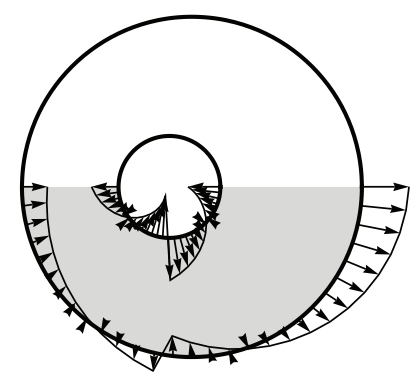

(c)

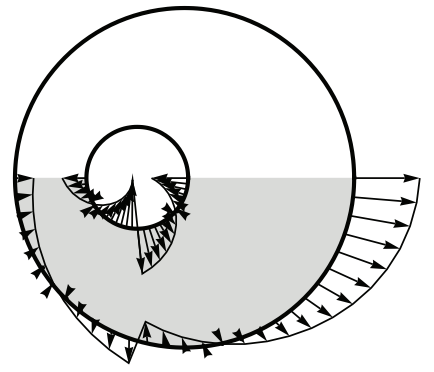

(e)

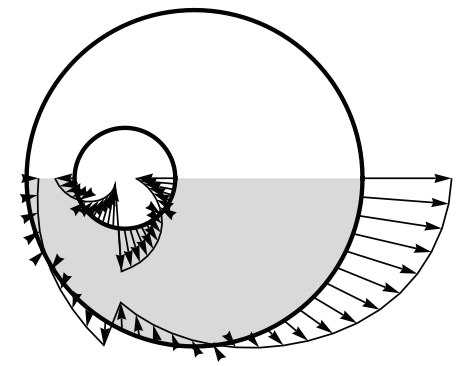

(g)

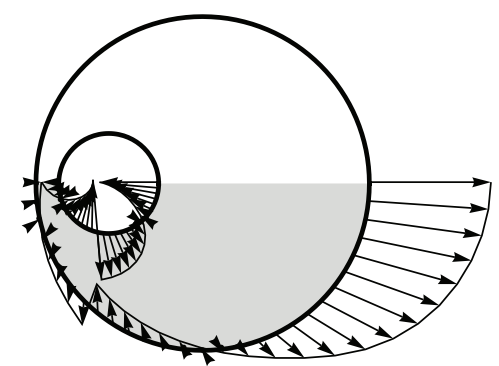

(i)

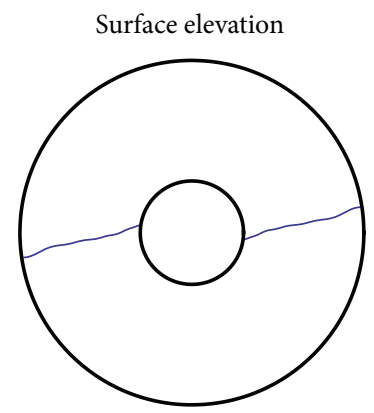

(b)

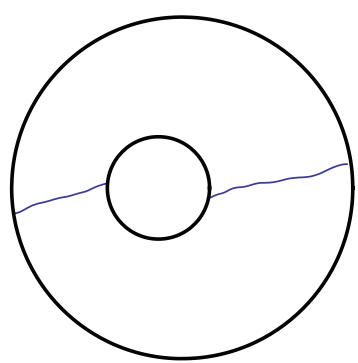

(d)

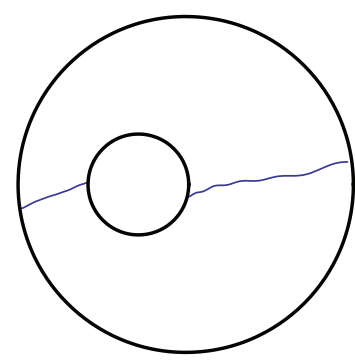

(f)

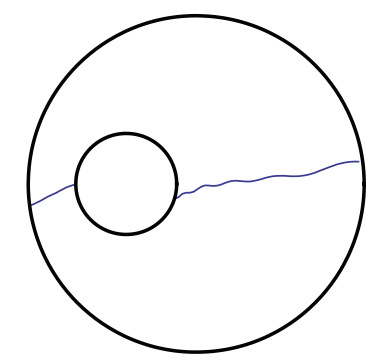

(h)

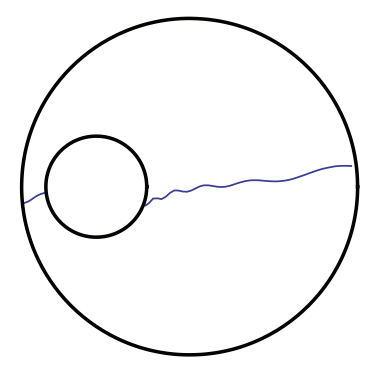

(j)

FIGURE 7: Typical pressure distributions acting on the tank wall and free surface elevation. 
surface elevation, for selected geometries $\left(r_{1}=0.3 ; r_{2}=1\right.$; $e=0.2,0.4,0.6,0.8)$, at selected time, $t=0.25 \mathrm{~s}$, and input time, $t_{0}=0.01 \mathrm{~s}$, specifically chosen to clearly illustrate the effects of tube configuration.

\section{Conclusion}

Reducing the sloshing and consequent undesired results to both environmental damages and economic losses force is the main purpose of the presented paper. In order to reach the system stability under transverse sloshing a new configuration is presented based on eccentric overall hole. An analytic approach based on the linearized theory of surface gravity waves in conjunction with the powerful conformal mapping technique is employed to investigate the transient twodimensional sloshing dynamic response of a perfect liquid partially filling a nondeformable horizontal circular eccentric annular tank. A detailed parametric study for examining the influence of the tube eccentricity and tube radius ratio on the hydrodynamic response is performed. In order to examine the performance of the designed configuration in suppressing the sloshing the system response under two separate excitations is evaluated: a ramp-step excitation encountered in a road turning maneuver as well as real seismic event is used to simulate the lateral acceleration excitation based on Laplace transform technique and Durbin's numerical inversion pattern. The effects of excitation input time, eccentricity, and radii ratio on the hydrodynamic responses and suppression of the induced destabilizing lateral forces are examined. Some of the most important observations are summarized as follows.

(i) After reaching a steady acceleration, the lateral forces continue to oscillate around the equilibrium point without any damping (ideal liquid). As a result the induced vibrating forces under oscillatory excitations, especially for sudden accelerations, cannot be ignored.

(ii) The largest lateral force response oscillations are noted for maneuvers with the lowest input time (swift accelerations). Moreover, as the input time increases, effects of tube size and/or configuration on the force response progressively decrease.

(iii) As the radius ratio increases the free surface length decreases and as a result $F_{s}$ decreases.

(iv) By increasing the eccentricity constant $e$ the pressure on the tank wall increases which is a result of increasing the liquid motion ability. Also increasing the radius ratio simultaneously, the effect of eccentricity on pressure profile shrinks (increases) because the free surface area lessens.

In addition, the validation of the presented model is evaluated by comparing the results with those obtained from recent models and commercial FEM software package.

\section{Conflict of Interests}

The authors declare that there is no conflict of interests regarding the publication of this paper.

\section{Acknowledgment}

The authors gratefully acknowledge the financial support of the Islamic Azad University, Firoozkooh Branch (Iran), in funding the work described here.

\section{References}

[1] R. A. Ibrahim, Liquid Sloshing Dynamics: Theory and Applications, Cambridge University Press, New York, NY, USA, 2nd edition, 2005.

[2] A. E. P. Veldman, J. Gerrits, R. Luppes, J. A. Helder, and J. P. B. Vreeburg, "The numerical simulation of liquid sloshing on board spacecraft," Journal of Computational Physics, vol. 224, no. 1, pp. 82-99, 2007.

[3] R. Monti, Physics of Fluids in Microgravity, CRC Press, Boca Raton, Fla, USA, 1st edition, 2002.

[4] B. N. Antar and V. S. Nuotio-Antar, Fundamentals of Low Gravity Fluid Dynamics and Heat Transfer, CRC Press, Boca Raton, Fla, USA, 1st edition, 1994.

[5] C. Hubert, "Behavior of spinning space vehicles with onboard liquids," in Proceedings of the Flight Mechanics Symposium, pp. 1-14, NASA, 2003.

[6] J. A. Romero, O. Ramírez, J. M. Fortanell, M. Martinez, and A. Lozano, "Analysis of lateral sloshing forces within road containers with high fill levels," Proceedings of the Institution of Mechanical Engineers D: Journal of Automobile Engineering, vol. 220, no. 3, pp. 302-312, 2006.

[7] L. Dai and L. Xu, "A numerical scheme for dynamic liquid sloshing in horizontal cylindrical containers," Proceedings of the Institution of Mechanical Engineers, Part D: Journal of Automobile Engineering, vol. 220, no. 7, pp. 901-918, 2006.

[8] C. H. Wu and B. F. Chen, "Sloshing waves and resonance modes of fluid in a 3D tank by a time-independent finite difference method," Ocean Engineering, vol. 36, no. 6-7, pp. 500-510, 2009.

[9] G.X. Wu, "Second-order resonance of sloshing in a tank," Ocean Engineering, vol. 34, no. 17-18, pp. 2345-2349, 2007.

[10] D. Liu and P. Lin, “Three-dimensional liquid sloshing in a tank with baffles," Ocean Engineering, vol. 36, no. 2, pp. 202-212, 2009.

[11] B. Budiansky, "Sloshing of liquids in circular canals and spherical tanks," Journal of the Aerospace Sciences, vol. 27, pp. 161-173, 1960.

[12] J. L. McCarty and D. Stephens, Investigation of the Natural Frequencies of Fluids in Spherical and Cylindrical Tanks, National Aeronautics and Space Administration, Washington, DC, USA, 1960.

[13] N. N. Moiseev and A. A. Petrov, "The calculation of free oscillations of a liquid in a motionless container," Advances in Applied Mechanics, vol. 9, pp. 91-154, 1966.

[14] P. McIver, "Sloshing frequencies for cylindrical and spherical containers filled to an arbitrary depth," Journal of Fluid Mechanics, vol. 201, pp. 243-257, 1989.

[15] N. Kobayashi, T. Mieda, H. Shibata, and Y. Shinozaki, "Study of the liquid slosh response in horizontal cylindrical tanks," Journal of Pressure Vessel Technology-Transactions of the ASME, vol. 111, no. 1, pp. 32-38, 1989.

[16] D. V. Evans and C. M. Linton, "Sloshing frequencies," Quarterly Journal of Mechanics and Applied Mathematics, vol. 46, no. 1, pp. 71-87, 1993. 
[17] G. Popov, S. Sankar, T. S. Sankar, and G. H. Vatistas, "Dynamics of liquid sloshing in horizontal cylindrical road containers," Proceedings of the Institution of Mechanical Engineers C: Journal of Mechanical Engineering Science, vol. 207, no. 2, pp. 399-406, 1993.

[18] S. Papaspyrou, S. A. Karamanos, and D. Valougeorgis, "Response of half-full horizontal cylinders under transverse excitation," Journal of Fluids and Structures, vol. 19, no. 7, pp. 985-1003, 2004.

[19] S. Papaspyrou, D. Valougeorgis, and S. A. Karamanos, "Sloshing effects in half-full horizontal cylindrical vessels under longitudinal excitation," Journal of Applied Mechanics, Transactions ASME, vol. 71, no. 2, pp. 255-265, 2004.

[20] S. A. Karamanos, L. A. Patkas, and M. A. Platyrrachos, "Sloshing effects on the seismic design of horizontal-cylindrical and spherical industrial vessels," Journal of Pressure Vessel Technology-Transactions of the ASME, vol. 128, no. 3, pp. 328340, 2006.

[21] L. A. Patkas and S. A. Karamanos, "Variational solutions for externally induced sloshing in horizontal-cylindrical and spherical vessels," Journal of Engineering Mechanics, vol. 133, no. 6, pp. 641-655, 2007.

[22] H. Zhou, J. F. Li, and T. S. Wang, "Simulation of liquid sloshing in curved-wall containers with arbitrary Lagrangian-Eulerian method," International Journal for Numerical Methods in Fluids, vol. 57, no. 4, pp. 437-452, 2008.

[23] A. A. Lakis, G. Bursuc, and M. H. Toorani, "Sloshing effect on the dynamic behavior of horizontal cylindrical shells," Nuclear Engineering and Design, vol. 239, no. 7, pp. 1193-1206, 2009.

[24] A. Gedikli and M. E. Ergüven, "Evaluation of sloshing problem by variational boundary element method," Engineering Analysis with Boundary Elements, vol. 27, no. 9, pp. 935-943, 2003.

[25] L. Strandberg, "Lateral stability of road tankers," VTI Report No. 138A, National Road and Traffic Research Institute, Linköping, Sweden, 1978.

[26] H. N. Abramson, "Slosh suppression," NASA Technical Report SP-8031, National Aeronautics and Space Administration, 1969.

[27] J. R. Cho, H. W. Lee, and K. W. Kim, "Free vibration analysis of baffled liquid-storage tanks by the structural-acoustic finite element formulation," Journal of Sound and Vibration, vol. 258, no. 5, pp. 847-866, 2002.

[28] I. Gavrilyuk, I. Lukovsky, Y. Trotsenko, and A. Timokha, "Sloshing in a vertical circular cylindrical tank with an annular baffle. I. Linear fundamental solutions," Journal of Engineering Mathematics, vol. 54, no. 1, pp. 71-88, 2006.

[29] K. C. Biswal, S. K. Bhattacharyya, and P. K. Sinha, "Nonlinear sloshing in partially liquid filled containers with baffles," International Journal for Numerical Methods in Engineering, vol. 68, no. 3, pp. 317-337, 2006.

[30] K. Modaressi-Tehrani, S. Rakheja, and I. Stiharu, "Threedimensional analysis of transient slosh within a partly-filled tank equipped with baffles," Vehicle System Dynamics, vol. 45, no. 6, pp. 525-548, 2007.

[31] A. Maleki and M. Ziyaeifar, "Sloshing damping in cylindrical liquid storage tanks with baffles," Journal of Sound and Vibration, vol. 311, no. 1-2, pp. 372-385, 2008.

[32] S. Chantasiriwan, "Modal analysis of free vibration of liquid in rigid container by the method of fundamental solutions," Engineering Analysis with Boundary Elements, vol. 33, no. 5, pp. 726-730, 2009.
[33] S. M. Hasheminejad and M. Aghabeigi, "Liquid sloshing in halffull horizontal elliptical tanks," Journal of Sound and Vibration, vol. 324, no. 1-2, pp. 332-349, 2009.

[34] G. R. Yan, S. Rakheja, and K. Siddiqui, "Baffle design analysis for a road tanker: transient fluid slosh approach," SAE International Journal of Commercial Vehicles, vol. 1, no. 1, pp. 397-405, 2009.

[35] G. R. Yan, S. Rakheja, and K. Siddiqui, "Analysis of transient fluid slosh in partly-filled tanks with and without baffles: part 1-model validation," International Journal of Heavy Vehicle Systems, vol. 17, no. 3-4, pp. 359-379, 2010.

[36] P. Pal, "Slosh dynamics of liquid-filled rigid containers: twodimensional meshless local Petrov-Galerkin approach," Journal of Engineering Mechanics, vol. 138, no. 6, pp. 567-581, 2012.

[37] O. M. Faltinsen and A. N. Timokha, "A multimodal method for liquid sloshing in a two-dimensional circular tank," Journal of Fluid Mechanics, vol. 665, pp. 457-479, 2010.

[38] H. Takahara and K. Kimura, "Frequency response of sloshing in an annular cylindrical tank subjected to pitching excitation," Journal of Sound and Vibration, vol. 331, no. 13, pp. 3199-3212, 2012.

[39] B. Robu, L. Baudouin, and C. Prieur, "Active vibration control of a fluid/plate system using a pole placement controller," International Journal of Control, vol. 85, no. 6, pp. 684-694, 2012.

[40] A. N. Williams and X. Wang, "Nonlinear transient wave motions in base-excited rectangular tanks," Journal of Fluids and Structures, vol. 6, no. 4, pp. 471-491, 1992.

[41] H. Akyildiz, "A numerical study of the effects of the vertical baffle on liquid sloshing in two-dimensional rectangular tank," Journal of Sound and Vibration, vol. 331, no. 1, pp. 41-52, 2012.

[42] J. H. Jung, H. S. Yoon, C. Y. Lee, and S. C. Shin, "Effect of the vertical baffle height on the liquid sloshing in a threedimensional rectangular tank," Ocean Engineering, vol. 44, pp. 79-89, 2012.

[43] C. Wu, O. M. Faltinsen, and B. Chen, "Numerical study of sloshing liquid in tanks with baffles by time-independent finite difference and fictitious cell method," Computers \& Fluids, vol. 63, pp. 9-26, 2012.

[44] M. Ali Goudarzi and S. Reza Sabbagh-Yazdi, "Investigation of nonlinear sloshing effects in seismically excited tanks," Soil Dynamics and Earthquake Engineering, vol. 43, pp. 355-365, 2012.

[45] O. M. Faltinsen, O. F. Rognebakke, and A. N. Timokha, "Classification of three-dimensional nonlinear sloshing in a squarebase tank with finite depth," Journal of Fluids and Structures, vol. 20, no. 1, pp. 81-103, 2005.

[46] A. Marsh, M. Prakash, E. Semercigil, and Ö. F. Turan, "A study of sloshing absorber geometry for structural control with SPH," Journal of Fluids and Structures, vol. 27, no. 8, pp. 1165-1181, 2011.

[47] E. Askari, F. Daneshmand, and M. Amabili, "Coupled vibrations of a partially fluid-filled cylindrical container with an internal body including the effect of free surface waves," Journal of Fluids and Structures, vol. 27, no. 7, pp. 1049-1067, 2011.

[48] H. Takahara, K. Hara, and T. Ishida, "Nonlinear liquid oscillation in a cylindrical tank with an eccentric core barrel," Journal of Fluids and Structures, vol. 35, pp. 120-132, 2012.

[49] S. Dutta and M. K. Laha, "Analysis of the small amplitude sloshing of a liquid in a rigid container of arbitrary shape using a low-order boundary element method," International Journal for Numerical Methods in Engineering, vol. 47, no. 9, pp. 1633-1648, 2000 . 
[50] J. D. Wang, S. H. Lo, and D. Zhou, "Liquid sloshing in rigid cylindrical container with multiple rigid annular baffles: free vibration," Journal of Fluids and Structures, vol. 34, pp. 138-156, 2012.

[51] F. B. Hildebrand, Advanced Calculus for Applications, Prentice Hall, Upper Saddle River, NJ, USA, 2nd edition, 1976.

[52] S. Mitra, P. P. Upadhyay, and K. P. Sinhamahapatra, "Slosh dynamics of inviscid fluids in two-dimensional tanks of various geometry using finite element method," International Journal for Numerical Methods in Fluids, vol. 56, no. 9, pp. 1625-1651, 2008.

[53] M. Salem, Rollover stability of partially filled heavy-duty elliptical tankers using trammel pendulums to simulate fluid sloshing [Ph.D. thesis], West Virginia University, Morgantown, WVa, USA, 2000. 

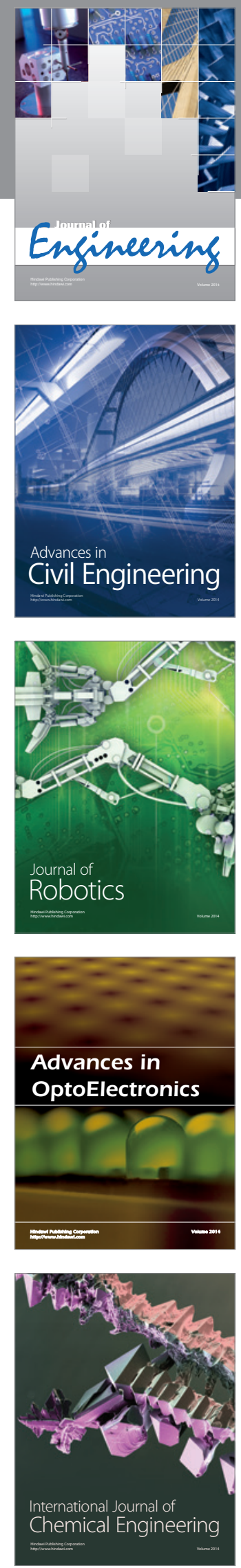

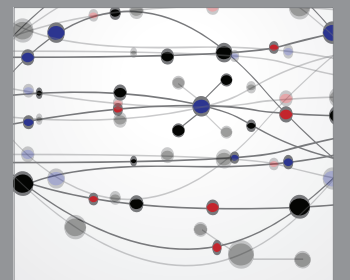

The Scientific World Journal
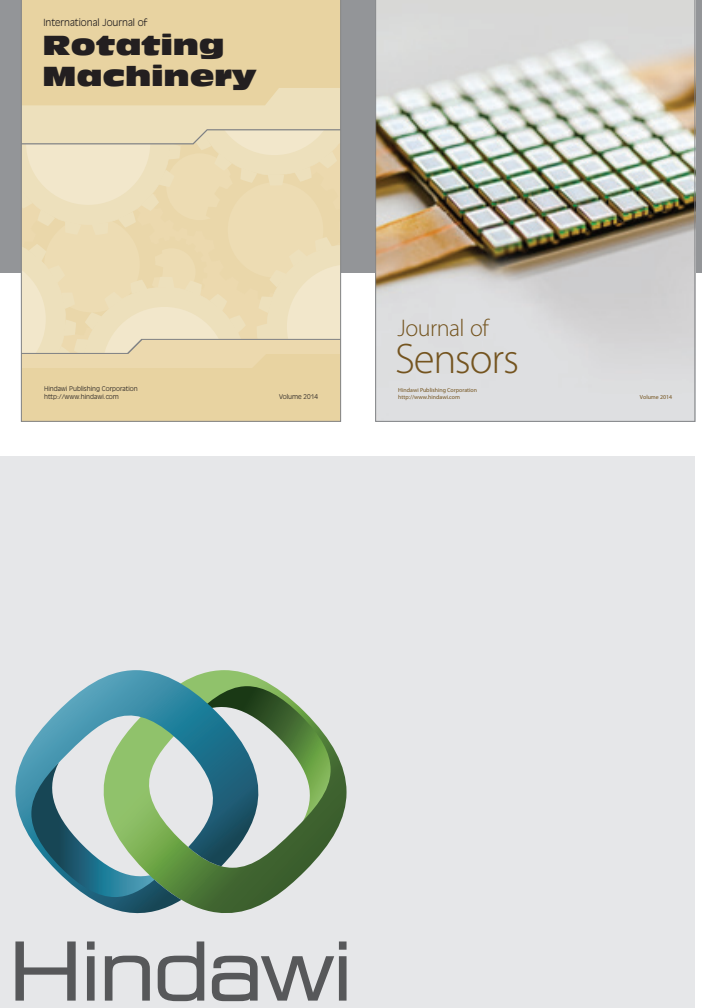

Submit your manuscripts at http://www.hindawi.com
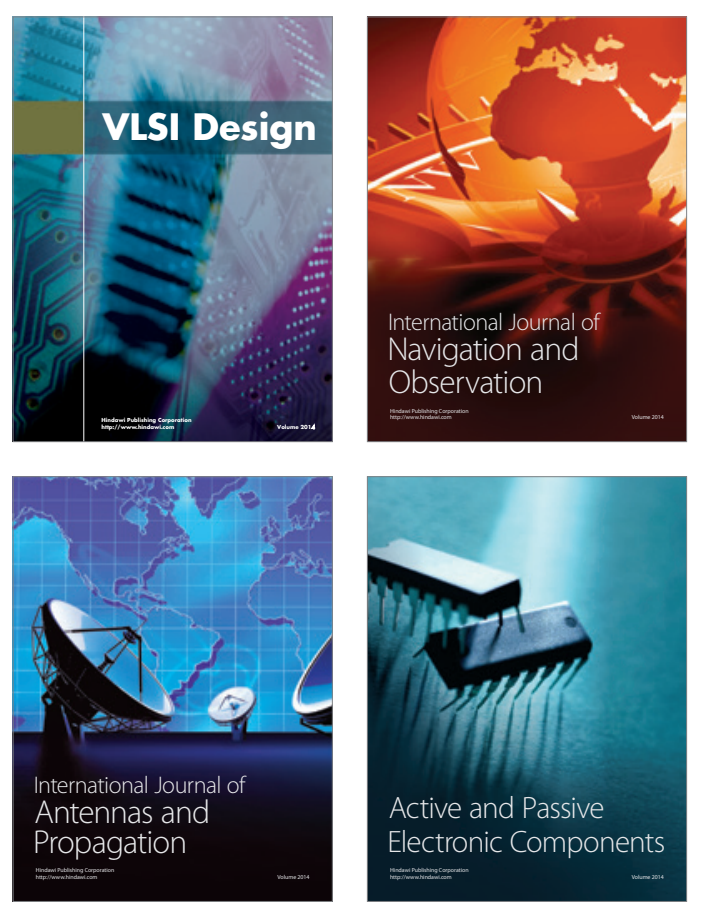
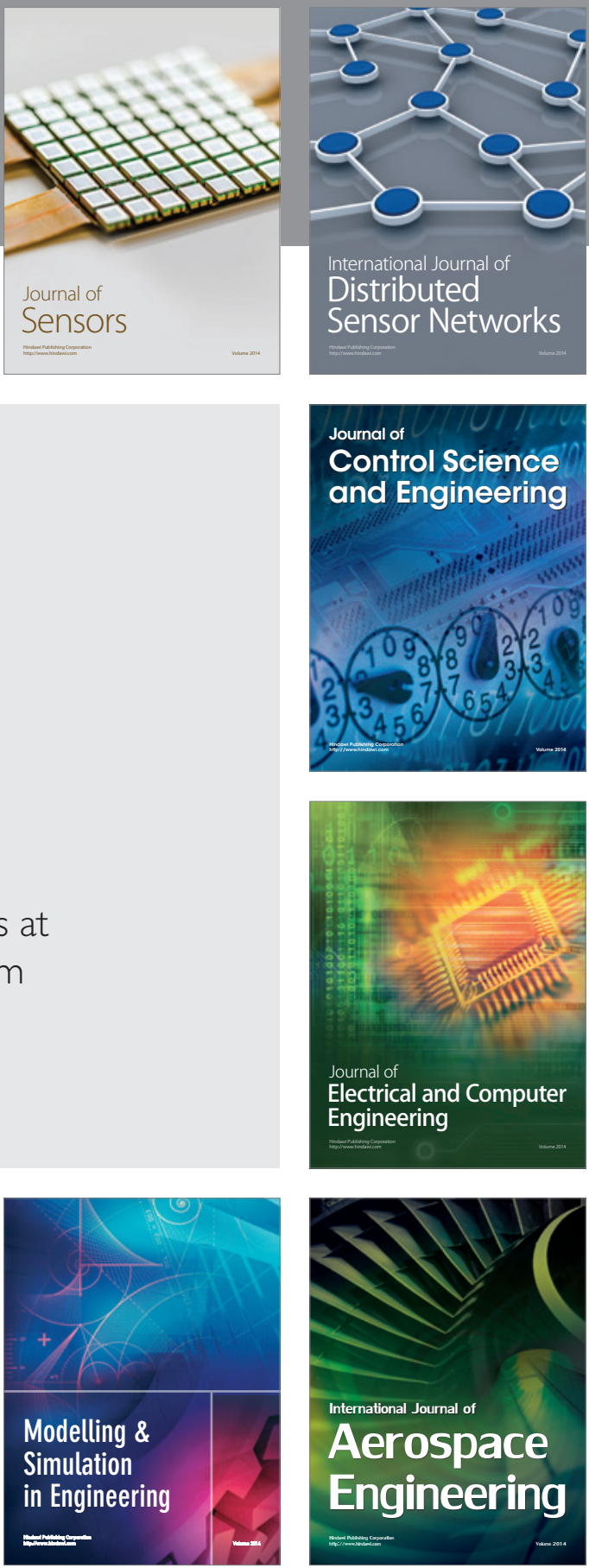

Journal of

Control Science

and Engineering
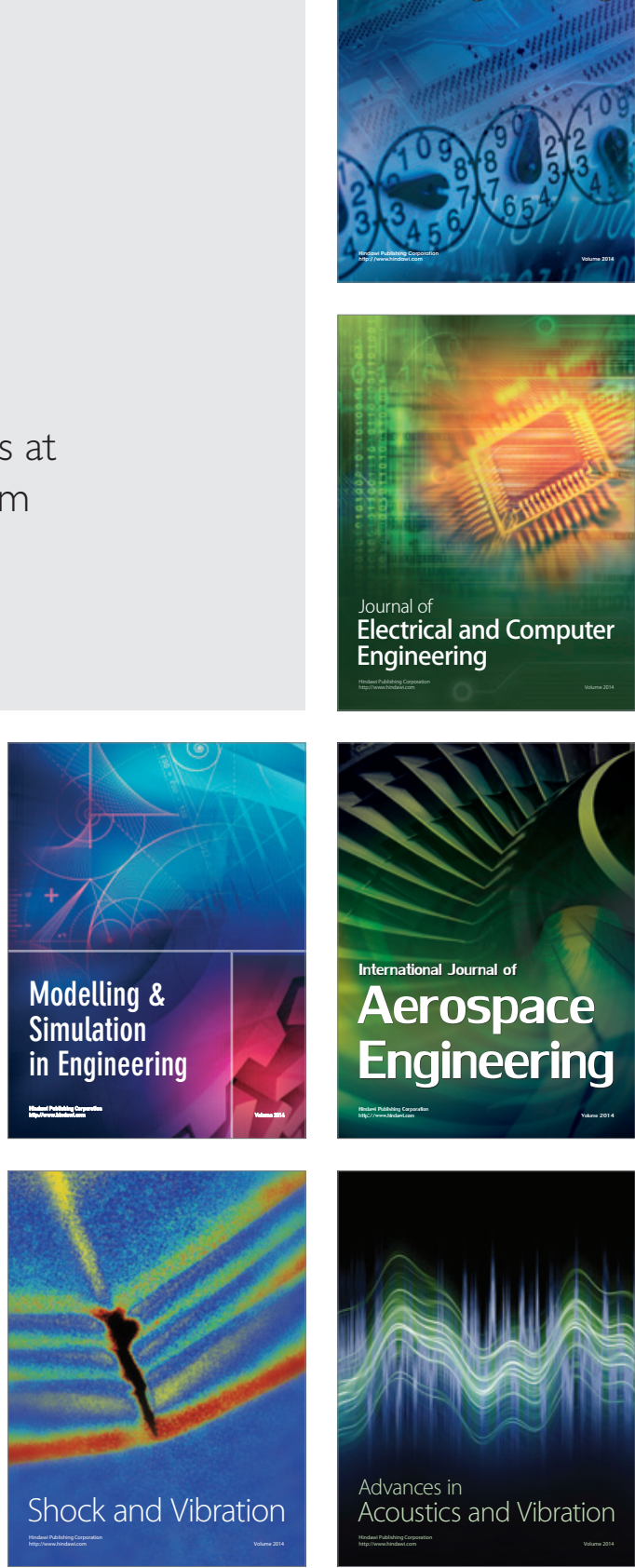